\title{
Overall and GTV subvolumes tumour control probability (TCP) for head and neck cancer treated by 3D-IMRT with inhomogeneous dose distribution
}

\author{
Leszek Hawrylewicz' , Bogusław Maciejewski², Klaus Rudiger Trott ${ }^{5}$, Andrzej Tukiendorf4 \\ Leszek Miszczyk², Magdalena Markowska ${ }^{3}$
}

\footnotetext{
${ }^{1}$ Department RT Planning, M. Skłodowska-Curie National Research Institute of Oncology, Gliwice Branch, Poland ${ }^{2}$ Department Radiotherapy, M. Skłodowska-Curie National Research Institute of Oncology, Gliwice Branch, Poland ${ }^{3}$ Division Research Programmes, M. Skłodowska-Curie National Research Institute of Oncology, Gliwice Branch, Poland ${ }^{4}$ Faculty of Health Sciences, Department of Public Health Medical University, Wroclaw, Poland ${ }^{5}$ Klinik und Poliklinik für Strahlentherapie der Technischen Universität München, Germany
}

Introduction. In this study, an original model has been developed to estimate the real TCP that is a product of the TCPS calculated for GTV subvolumes of head and neck cancer based on 3D-IMRT dose planning.

Material and methods. Retrospective pilot group consist of 16 cases of oropharyngeal cancer in stage T1-2N0 previously treated with 3D-IMRT with at least 3-year follow-up. The total dose (TD) was 60-70 Gy in 2.0 Gy fractions delivered over 42-49 days. Within GTV two subvolumes were marked out: SVA with the planned 100\% TD, and underdosed (90-95\%) SVB. The TCP for both was calculated using the original formula developed by Withers and Maciejewski.

Results. During 3-year follow-up, 8 local recurrences (LR) occurred. In about 70\% of SVB "dose cold spots" encompassed more than $50 \%$ GTV volume. This resulted in the TCP SVB $_{\text {B }}$ decrease to $60 \%$. Thus, the real overall TCP was much lower than a priori predicted, and in these cases local recurrences occurred.

Discussion. Both cold spot SVB volumes and their dose deficit strongly correlated with a high risk of LR.

Conclusions. In conclusion the magnitude of dose deficit and the size of cold subvolume within GTV have an independent negative impact on real TCP and demand dose re-planning.

Key words: 3D-IMRT planning, cold spots within GTV, estimates of partial TCPs within GTV subvolumes

\section{Introduction}

Tumour cure using radiotherapy requires the sterilisation of all tumour stem cells. A single surviving functional tumour stem cell has a high probability of causing local tumour recurrence [1]. With increasing radiation dose to the GTV, the number of surviving tumour stem cells decreases exponentially, leading to a dose-dependent rise of tumour control probability (TCP) which follows a Poisson function of the probability that no tumour stem cell survived in the GTV. The logical consequence of this mechanism is that only two factors determine the dependence of the TCP on tumour dose (TD), but only in the case of homogeneous dose distribution in the GTV:

- the absolute number of tumour stem cells (which is related to the absolute tumour volume, the stem cell fraction among all tumour cells and the repopulation rate during the duration of the treatment);

\section{How to cite:}

Hawrylewicz L, Maciejewski B, Trott KR, Tukiendorf A, Miszczyk L, Markowska ML. Overall and GTV subvolumes tumour control probability (TCP) for head and neck cancer treated by 3D-IMRT with inhomogeneous dose distribution. NOWOTWORY J Oncol 2020; 70: 127-134. 
- the slope of the exponential decrease of the fraction of surviving tumour stem cells within the irradiated volume (which depends on the dose per fraction, the intrinsic tumour stem cell radiosensitivity, and which may also be influenced by micro-environmental factors).

The dependence of the curative dose (TCP-50) on the tumour volume has been investigated in experimental tumours (in particular by Suit [2] and by Guttenberger [3]) and in clinical studies, (e.g. Maciejewski et al. [4], Dubben et al. [5] and Magee et al. [6]). The analysis of these data suggests that a ten-fold difference in the absolute number of tumour stem cells between tumours of the same type and T-stage (which may be due to differences in gross tumour volume, tumour stem cell fraction at the start of radiotherapy or accelerated repopulation during radiotherapy) may represent a difference in TCD-50 of around 7 Gy. However, the relationship between TCP and TCD-50 is much more complicated when the dose in the GTV is heterogeneously distributed. Theoretical calculations of the impact of dose inhomogeneity within the PTV/ GTV have been published, yet little clinical evidence to support these calculations has been presented so far.

When the 3D-IMRT was introduced into daily practice it became obvious that a dose gradient within the target leads to non-uniform dose distribution in the tumour volume. Tome and Fowler [7, 8] calculated an increase in the TCP for tumour subvolumes boosted to higher dose, and TCP loss within under-dosed sub-volumes ("cold spots"). It was concluded that the clinical impact of a dose deficit would depend not only on the magnitude of the deficit but also on the size of subvolume [9-11].

More than 15 years ago, Withers and Maciejewski developed a radiobiological model for changes in the TCP estimates for subvolumes and their dependence on initial tumour stem cell number represented by the size of the respective subvolumes and the total doses delivered [unpublished]. However, at that time, 2D radiotherapy with homogenous dose distribution within the target was the standard and dose differences in the GTV subvolumes were not a problem. Nowadays, 3D-IMRT with heterogeneous dose distribution within the GTV is widely used, which may impact on the TCP [12-15].

\section{Material and methods}

\section{Dose planning data}

For the present study, a pilot set of 16 consecutive 3D-IMRT treatment plans for T1-T2NOMO, sq.C.c of the oral cavity, oropharynx and supraglottic larynx, all with at least 3-year follow-up and with apparent inhomogeneous dose distribution within the GTV were selected from the treatment planning data bank in our institution. Inhomogeneous dose distribution was defined as sub-volumes larger than 5\% of the GTV in which the total dose was reduced by $>5 \%$. Treatment plans with homogenous dose distribution D100 or D95 covering the whole GTV were not taken into account.

\section{Radiotherapy}

For all 16 patients, the 3D - treatment plans and DVHs were developed using the Eclipse Planning System (version 8.6 or 13, Varian). Using the Clinac 2300 accelerator with 120 MLC and 3D-IMRT technique, conventional 2.0 Gy daily fractions were delivered 5 days a week to a total dose ranging from $60 \mathrm{~Gy}$ in 42 days to $70 \mathrm{~Gy}$ in 48 days. There were no extensions of overall treatment time, and therefore the time factor is not considered in the analysis.

\section{Tumour volume measurements}

For the purpose of this study, tumour volumes were estimated from the data bank of the CT/MRI sequential scans spaced by 2-3 $\mathrm{mm}$ as proposed by Johnson et al. [11]. The primary tumour was outlined in each scan at the TPS workstation and the tumour volume was calculated by a computer-based analysis system. The primary tumour volume was defined as GTV, which ranged from $2.5 \mathrm{~cm}^{2}$ to $29.2 \mathrm{~cm}^{2}$.

For the purpose of the present analysis, the tumour volume was subdivided into two subvolumes:

- SVA - the volume of the GTV covered by $100 \%$ isodoses of the planned total dose (D100);

- SVB - the volume of the GTV covered by on average 90-95\% of the planned total dose (D90-95). The total dose for this subvolume was converted into biologically normalised total dose (BNTD) if given in 2.0 Gy fractions using the L-Q model with = $10 \mathrm{~Gy}$.

\section{Initial stem cell number (K)}

Following the assumptions made by McBride and Withers [9], a tumour of $1 \mathrm{~cm}$ in diameter $\left(\mathrm{v}=0.52 \mathrm{~cm}^{3}\right)$ was assumed as a standard volumetric unit, containing $10^{9}$ cells $[9,16,17]$, among which $1 \%$ possesses stem cell potential $\left(10^{7}\right.$ tumour stem cells). Then, the initial stem cell number in each specific primary tumour volume $\left(\mathrm{V}_{\mathrm{i}}\right)$ would be:

$$
K_{i}=107 \times\left(V_{i} / 0.52\right)
$$

Stem cell numbers in subvolumes SVA and SVB were calculated using the same equation [1], using SVA and SVB volumetric parameters.

\section{Tumour cure probability (TCP)}

The relationship between the number of surviving tumour stem cells $\left(K_{S}\right)$, tumour volume $\left(V_{i}\right)$ and total dose $\left(T_{i}\right)$ approximates simple Poisson statistics [18, 19]:

$$
\mathrm{TCP}=\exp (-\mathrm{K})
$$

where $K_{i}$ is equal:

$$
\mathrm{K}_{\mathrm{s}}=\mathrm{K}_{\mathrm{i}} \times \mathrm{SF}_{\mathrm{s}}
$$


in which $\mathrm{K}_{\mathrm{i}}$ is initial stem cell number and $\mathrm{SF}_{\mathrm{S}}$ is the surviving fraction after the total dose (TD).

This equation can be rearranged as follows:

$$
\mathrm{TCP}=\exp \left(-\mathrm{K}_{\mathrm{i}} \times \mathrm{SF}_{\mathrm{S}}\right)
$$

Surviving fractions may be estimated using various methods, such as SF2.0 (surviving fraction after a dose of $2.0 \mathrm{~Gy}$ ), or effective $D_{10}$ which is the dose that reduces survival to $e^{-1}$ for a particular fraction regimen or the LQ model. These three methods are mainly used in experimental radiobiology, but are not very practical for daily clinical radiotherapy.

Mc Bride and Withers [9] suggested that the surviving fraction can more easily be determined in terms of $\mathrm{eD}_{10}$, i.e. the dose which reduces stem cell survival by one decade to $10 \%$. In our study this parameter was used. An approximate value for $\mathrm{eD}_{10}$ for $2.0 \mathrm{~Gy}$ fractions was suggested as about $7 \mathrm{~Gy}[1,9,16]$. Therefore using $\mathrm{eD}_{10}=7 \mathrm{~Gy}$, for a tumour treated with the total dose TD, the absolute number of surviving functional stem cells (not surviving fractions) would be reduced to $10-\mathrm{TD}_{\mathrm{i}} / \mathrm{eD} \mathrm{D}_{10}$.

Combining equations the subvolume $\mathrm{TCP}_{\mathrm{i}}$ can be calculated from equation:

$$
\mathrm{TCP}_{\mathrm{i}}=\exp \left[-\left(10^{7} \times\left(\mathrm{V}_{\mathrm{i}} / 0.52\right) \times\left(10-^{\left.\mathrm{TD}_{\mathrm{i}} / \mathrm{eD}_{10}\right)}\right]\right.\right.
$$

where $10^{7}$ is approximately the number of stem cells in a tumour $1 \mathrm{~cm}$ in diameter $\left(0.52 \mathrm{~cm}^{3}\right), V_{i}$ - is tumour subvolume, and $\mathrm{TD}_{\mathrm{i}}$ is the delivered total dose.

$\mathrm{TCP}_{i}$ values were calculated using the previously given parameters for the GTV and subvolumes A and B. Finally, the real TCP $\mathrm{RL}_{\mathrm{L}}$ was calculated as a product of the $T C P_{A}$ and the $T C P_{B}$ :

$$
\mathrm{TCP}_{\mathrm{RL}}=\mathrm{TCP}_{\mathrm{A}} \times \mathrm{TCP}_{\mathrm{B}}
$$

For all 3D-IMRT plans, $\mathrm{TCP}_{\mathrm{PL}}$ and $\mathrm{TCP}_{\mathrm{RL}}$ were compared and finally related with 3-year follow-up clinical results (local recurrence or disease-free survival).

\section{Clinical data}

After completing the results of $\mathrm{TCP}_{\mathrm{PL}}$, and $\mathrm{TCP} \mathrm{RL}_{\mathrm{RL}}$ calculations, they were compared with retrospective 3-year treatment outcomes of the selected 16 patients previously treated with 3D-IMRT. The outcome end-points, i.e. local tumour control (LTC) and local recurrence (LR) were considered. There was no incidence of distant metastases.

\section{Results}

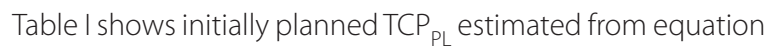
[5] for the data taken from treatment planning charts of the group of 16 cases. Dose planning and delivery had been prescribed by individual radiation oncologists generally based on the T stage criterion, even though tumour volumes differed by about 10 times $\left(2.5 \mathrm{~cm}^{3}-29.2 \mathrm{~cm}^{3}\right)$. Although there were no extensions in overall treatment time and the standard fraction of 2.0 Gy was given regularly, 5 days a week, in hindsight, the choice of the total doses for some cases seems illogical, e.g. TD of 70 Gy was given to $4.55 \mathrm{~cm}^{3}$ (pt. no. 2) whereas the tumour volume 2.5 larger (case no. 11) received only 60 Gy and the largest one in this series (case no. 16) received $63 \mathrm{~Gy}$.

Nevertheless, except for two cases (no. 7 and no. 16), estimates of the planned TCP $P_{P L}$ are within an acceptable range and predicted a high probability of local tumour control.

The analysis of the impact of the subvolumes $A$ and $B$ within GTV on estimated values of the TCP (tab. II) shows that $\mathrm{TCP}_{\mathrm{A}}$ estimated for SVA were generally very high. However they do not correlate with the incidence of local recurrence.

In contrast with SVA, the size of subvolumes SVB, and derived NTD and partial TCP $_{B}$ values had a strong impact on the estimated real TCP values, which were decreased by $3-74 \%$ compared to the initial $\mathrm{TCP}_{\mathrm{PL}}$ calculated from the treatment plans. Three-dimensional least square $(20,21)$ planes for dose-volume-TCP relationship are presented in figure 1.

The spatial distribution of these three parameters estimated prior to therapy appear to be of little use in predicting the risk of local recurrence (fig. 1 a). The correlation was even weaker when the SVA was analysed (fig. 1 b). Local recurrence was observed in patients who received the prescribed TD. In contrary, figure 1 c shows a significant impact of "cold" dose in SVB on $\mathrm{TCP}_{\mathrm{B}^{\prime}}$ which was particularly strong when, within

Table I. Planned TCP values for all 16 patients and gross tumour volume (GTV) and the calculated number of tumour stem cells and prescribed total dose (NTDp). Black dots indicate that a local tumour recurrence occurred during 3-year follow-up

\begin{tabular}{|lccccc}
\hline $\begin{array}{l}\text { Pts } \\
\text { No }\end{array}$ & $\begin{array}{c}\text { T } \\
\text { Stage }\end{array}$ & $\begin{array}{c}\text { VOL. (GTV) } \\
\mathrm{cm}^{3}\end{array}$ & $\begin{array}{c}\text { Log }_{10} \\
\text { K p }\end{array}$ & $\begin{array}{c}\text { NTD p } \\
\text { izobio Gy }\end{array}$ & $\begin{array}{c}\text { Planned } \\
\text { TCP } p\end{array}$ \\
\hline 1 & $\mathrm{~T} 1$ & 2.5 & 7.7 & 60 & $\sim 88 \%$ \\
\hline 2 & $\mathrm{~T} 1$ & 4.55 & 7.95 & 70 & $\sim 99 \%$ \\
\hline 3 & $\mathrm{~T} 1$ & 5.4 & 8 & 60 & $\sim 77 \% \bullet$ \\
\hline 4 & $\mathrm{~T} 1$ & 5.6 & 8 & 60 & $\sim 77 \% \bullet$ \\
\hline 5 & $\mathrm{~T} 2$ & 6.2 & 8.1 & 60 & $\sim 71 \% \bullet$ \\
\hline 6 & $\mathrm{~T} 2$ & 6.2 & 8.1 & 60 & $\sim 71 \%$ \\
\hline 7 & $\mathrm{~T} 2$ & 8.1 & 8.2 & 60 & $\sim 65 \% \bullet$ \\
\hline 8 & $\mathrm{~T} 2$ & 9.5 & 8.3 & 66 & $\sim 93 \%$ \\
\hline 9 & $\mathrm{~T} 2$ & 11.0 & 8.33 & 66 & $\sim 92 \%$ \\
\hline 10 & $\mathrm{~T} 2$ & 11.5 & 8.34 & 66 & $\sim 92 \%$ \\
\hline 11 & $\mathrm{~T} 2$ & 12.5 & 8.4 & 60 & $\sim 51 \% \bullet$ \\
\hline 12 & $\mathrm{~T} 2$ & 14.0 & 8.43 & 66 & $\sim 90 \% \bullet$ \\
\hline 13 & $\mathrm{~T} 2$ & 15.0 & 8.46 & 70 & $\sim 97 \% \bullet$ \\
\hline 14 & $\mathrm{~T} 2$ & 19.0 & 8.56 & 66 & $\sim 87 \%$ \\
\hline 15 & $\mathrm{~T} 2$ & 22.0 & 8.63 & 70 & $\sim 96 \%$ \\
\hline 16 & $\mathrm{~T} 2$ & 29.2 & 8.74 & 63 & $58 \% \bullet$ \\
\hline
\end{tabular}


Table II. Estimates of the TCP for subvolumes SVA and SVB within GTV, and realistic TCP as a product of both estimates (SVA is covered by TD100 and SVB by TD90-95). Black dots indicate that a local tumour recurrence occurred during 3-year follow-up

\begin{tabular}{|c|c|c|c|c|c|c|c|c|c|}
\hline \multirow{2}{*}{$\begin{array}{l}\text { Pts } \\
\text { No }\end{array}$} & \multicolumn{3}{|c|}{ SUBVOLUME A } & \multicolumn{3}{|c|}{ SUBVOLUME B $\left(\mathrm{V}_{90-95}\right)$} & \multirow{2}{*}{$\begin{array}{c}\operatorname{TCP}_{\text {ESTIM }} \\
\left(\mathrm{TCP}_{A} \times \mathrm{TCP}_{B}\right)\end{array}$} & \multirow[b]{2}{*}{$\mathrm{TCP}_{\mathrm{P}}-\mathrm{TCP}_{\mathrm{E}}$} & \multirow{2}{*}{$\begin{array}{c}\text { 3-year } \\
\text { follow-up }\end{array}$} \\
\hline & $\% \mathrm{VOL}_{\mathrm{GTV}}$ & $\begin{array}{l}\mathrm{NTD}_{\mathrm{A}} \\
\text { izoGy }_{2.0}\end{array}$ & $\mathrm{TCP}_{\mathrm{A}}$ & $\% \mathrm{VOL}_{\mathrm{GTV}}$ & $\begin{array}{c}\mathrm{NTD}_{\mathrm{B}} \\
\text { izoGy }_{2.0}\end{array}$ & $\mathrm{TCP}_{\mathrm{B}}$ & & & \\
\hline 1 & $V_{48}$ & $60 \mathrm{~Gy}$ & $94 \%$ & $V_{52}$ & $56.8 \mathrm{~Gy}$ & $78 \%$ & $73 \%$ & $-15 \%$ & DFS \\
\hline 2 & $V_{73}$ & 70 Gy & $99.5 \%$ & $V_{27}$ & $61.1 \mathrm{~Gy}$ & $95 \%$ & $94 \%$ & $-5 \%$ & DFS \\
\hline 3 & $V_{43}$ & 60 Gy & $88 \%$ & $V_{57}$ & $55.8 \mathrm{~Gy}$ & $53 \%$ & $47 \%$ & $-41 \%$ & $\mathrm{LR} \bullet$ \\
\hline 4 & $V_{21}$ & 60 Gy & $94 \%$ & $V_{79}$ & $56.8 \mathrm{~Gy}$ & $52 \%$ & $49 \%$ & $-28 \%$ & $\mathrm{LR} \bullet$ \\
\hline 5 & $V_{6}$ & $60 \mathrm{~Gy}$ & $98 \%$ & $V_{94}$ & $56.7 \mathrm{~Gy}$ & $41 \%$ & $40 \%$ & $-31 \%$ & $\mathrm{LR} \bullet$ \\
\hline 6 & $V_{79}$ & $60 \mathrm{~Gy}$ & $78 \%$ & $V_{21}$ & $57.4 \mathrm{~Gy}$ & $86 \%$ & $67 \%$ & $-4 \%$ & DFS \\
\hline 7 & $V_{71}$ & 60 Gy & $76 \%$ & $\mathrm{~V}_{29}$ & $55.9 \mathrm{~Gy}$ & $62 \%$ & $47 \%$ & $-18 \%$ & $\mathrm{LR} \bullet$ \\
\hline 8 & $V_{82}$ & 66 Gy & $95 \%$ & $V_{18}$ & $63.2 \mathrm{~Gy}$ & $97 \%$ & $92 \%$ & $-3 \%$ & DFS \\
\hline 9 & $V_{5}$ & 66 Gy & $99 \%$ & $V_{95}$ & $62.4 \mathrm{~Gy}$ & $78 \%$ & $77 \%$ & $-15 \%$ & DFS \\
\hline 10 & $V_{5}$ & 66 Gy & $99 \%$ & $V_{95}$ & 60 Gy & $56 \%$ & $55 \%$ & $-37 \%$ & DFS \\
\hline 11 & $V_{80}$ & 60 Gy & $59 \%$ & $\mathrm{~V}_{20}$ & $56.3 \mathrm{~Gy}$ & $65 \%$ & $38 \%$ & $-13 \%$ & $\mathrm{LR} \bullet$ \\
\hline 12 & $V_{4}$ & 66 Gy & $99.5 \%$ & $V_{96}$ & $60.1 \mathrm{~Gy}$ & $51 \%$ & $50 \%$ & $-40 \%$ & $\mathrm{LR} \bullet$ \\
\hline 13 & $V_{40}$ & $70 \mathrm{~Gy}$ & $99 \%$ & $V_{60}$ & $56.5 \mathrm{~Gy}$ & $23 \%$ & $23 \%$ & $-74 \%$ & $\mathrm{LR} \bullet$ \\
\hline 14 & $V_{13}$ & $66 \mathrm{~Gy}$ & $98 \%$ & $V_{87}$ & $63.1 \mathrm{~Gy}$ & $73 \%$ & $71 \%$ & $-16 \%$ & DFS \\
\hline 15 & $V_{45}$ & 70 Gy & $98 \%$ & $V_{55}$ & $64.2 \mathrm{~Gy}$ & $85 \%$ & $83 \%$ & $-13 \%$ & DFS \\
\hline 16 & $V_{12}$ & 63 Gy & $94 \%$ & $V_{88}$ & $60.7 \mathrm{~Gy}$ & $35 \%$ & $33 \%$ & $-25 \%$ & $\mathrm{LR} \bullet$ \\
\hline
\end{tabular}

the GTV, the SVB was larger than the SVA. Therefore, the size of SVB and respective values of "cold"TDB, but not SVA and it parameters, substantially impacted on the decrease in the real TCP compared with the initially planned TCP. The real TCP $R$ strongly correlated with the incidence of local recurrence.

\section{Replanning of dose distribution in cases of large SVB}

During 3D radiotherapy planning, heterogeneous dose distribution within the target volume needs detailed searching for possible "cold spots" and "cold doses", not so much in the CTV and PTV but above all in the GTV. The treatment plan and the dose distribution should be revised by a mathematically simple calculation of the realistic TCP ${ }_{\mathrm{RL}}$ and compared with the conventionally determined TCP $P_{\mathrm{PL}}$. For this task we recommend using equation [6]. It is a simple and non-time-consuming procedure. If an unacceptable decrease in real $\mathrm{TCP}_{\mathrm{RL}}$ compared with the planned TCP $P_{P L}$ is found, the dose distribution within specified volumes needs to be corrected, which should lead to as uniform a dose distribution as possible at least in the GTV.

Table III shows an option of corrections of SVA and SVB and respective TDA and TDB for 16 cases previously listed in table II to achieve uniformly high $\mathrm{TCP}_{A R}$ and $\mathrm{TCP}_{B R}$ above $90 \%$, finally resulting in an increase in realistic $T C P_{R L}$. This exemplifies a way to minimise or even eliminate "cold spot"and "cold dose" in the GTV to get a real $T C P_{R L}$ close or equal to that originally planned $\left(T C P_{P L}\right)$.

\section{Discussion}

Many authors have emphasised that both tumour volume (TV) and tumour dose define tumour control probability (TCP) [5, 11-14, 17, 22]. Tumour stage $(T)$, however, fails to provide relia- ble information of tumour volume and TCP. Therefore tumour staging cannot replace measurement of tumour volumes. Even within one tumour stage, TV can vary considerably as shown in table I: In the group of T1-T2N0M0 treatment plans for oral cavity and oropharyngeal cancer investigated, there was a 10-fold difference in the TV.

Analysing a survey of cervix, breast, head and neck and melanoma clinical data Dubben et al. [5] produced a series of steep TCP-TV-curves. Because in our model study, 16 treatment plans were randomly chosen from our clinical data bank, we cannot explain why some small TV (case no. 2) were treated with 70 Gy whereas much larger TV received $60 \mathrm{~Gy}$. It was the individual choice of different radiation oncologists, who prescribed total doses according to the T-stages of tumours.

The absolute number of tumour stem cells has been shown to be proportional to the tumour volume in most rodent and human cancers (unless there are large necrotic volumes found $[1,2,11,12])$. For the purpose of our study, we assumed that $1 \%$ of tumour cells are tumour stem cells [9].

A local control rate of $90 \%$ results if on average $0.1 \mathrm{tu}$ mour stem cells survive, or in other words, if one in ten irradiated tumours contains one or more tumour stem cell. In a tumour with about $10^{9}$ tumour stem cells such as \#16, the stem cell surviving fraction has to be about $10^{-10}$ to achieve a local control rate of $90 \%$. Using $\mathrm{eD}_{10}$ of 7.0 Gy assumed in our model, it would require a total dose of approximately 70 Gy instead of the 63 Gy given to increase TCP from approximately $40 \%$ to $90 \%$. The observed local recurrence thus had to be expected. 


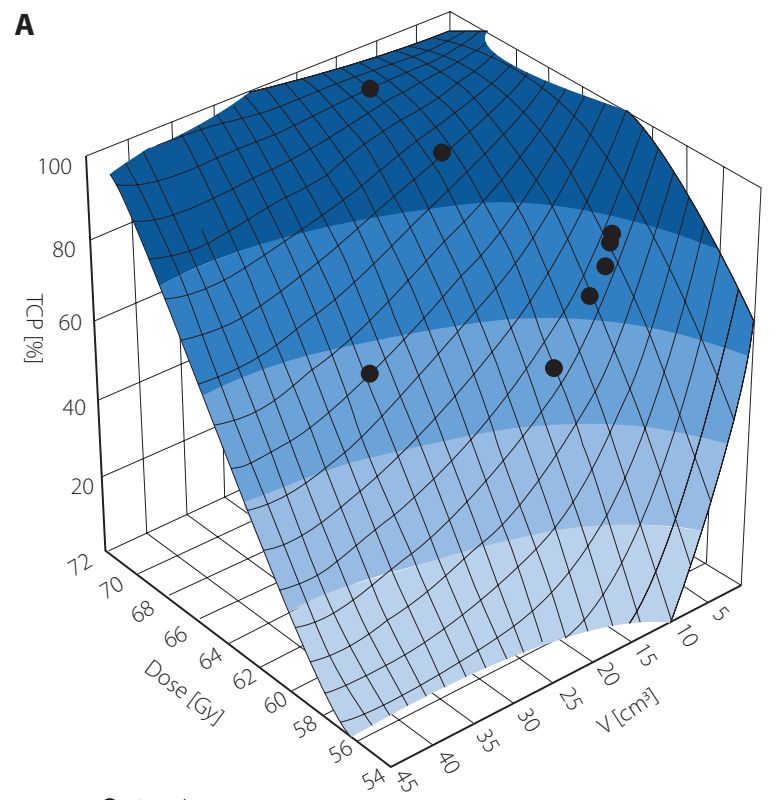

- Local resurance

TCP [\%]
B

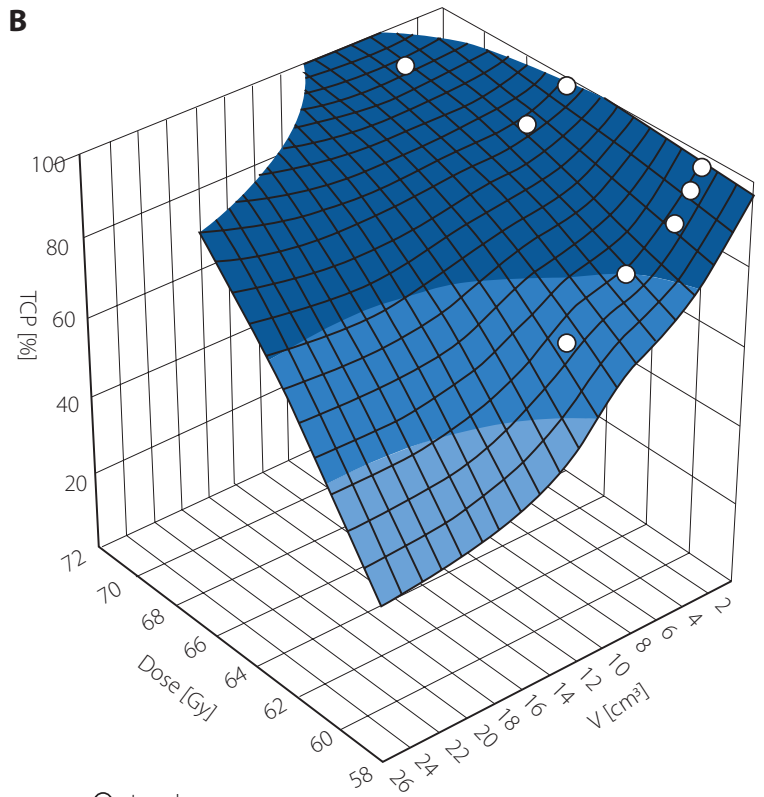

Local recurrence

TCP [\%]

$\square>80 \quad \square<80 \quad \square<60$

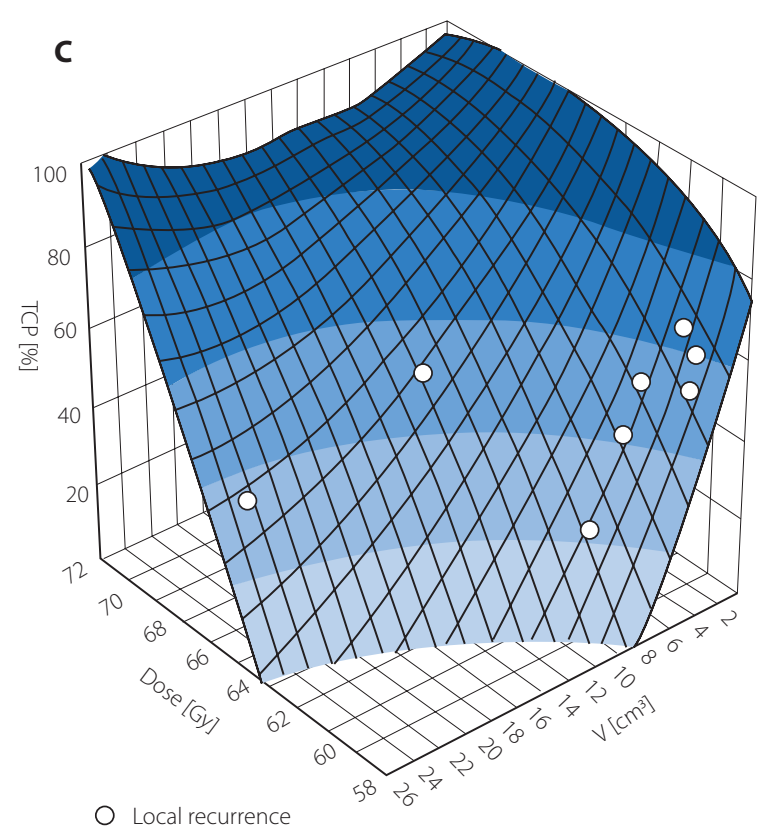

O Local recurrence

TCP [\%]

$\square>80 \quad \square<80 \quad \square<60 \quad \square<40 \quad<20$

Figure 1.3D-least square planes for dose-volume-TCP relationships: A - for planned parameters; B - for subvolume SVA; C - for subvolume SVB; black dots indicate local tumour recurrence occurred during 3-year follow-up

Table III. Examples of re-planning of dose distribution within the GTV subvolumes SVA and SVB in all patients to get similarly high TCPs in both, and also high realistic overall TCP

\begin{tabular}{|c|c|c|c|c|c|c|c|}
\hline \multirow{2}{*}{$\begin{array}{l}\text { Pts } \\
\text { No }\end{array}$} & \multicolumn{3}{|c|}{ SUBVOLUME $\mathrm{A} \rightarrow \mathrm{ACr}$} & \multicolumn{3}{|c|}{ SUBVOLUME B $\rightarrow \mathrm{BCr}$} & \multirow{2}{*}{$\left(\mathrm{TCP}_{\mathrm{ACr}} \times \mathrm{TCP}_{\mathrm{real}}\right.$} \\
\hline & $V_{A} \rightarrow V_{A R}$ & $\begin{array}{c}\mathrm{NTD}_{\mathrm{A}} \rightarrow \mathrm{NTD}_{\mathrm{AR}} \\
\left(\mathrm{izOGy}_{2.0}\right)\end{array}$ & $\mathrm{TCP}_{\mathrm{ACr}}$ & $\mathrm{V}_{\mathrm{B}} \rightarrow \mathrm{V}_{\mathrm{BR}}$ & $\begin{array}{c}\mathrm{NTD}_{\mathrm{B}} \rightarrow \mathrm{NTD}_{\text {BR }} \\
\quad\left(\mathrm{izoGy}_{2.0}\right)\end{array}$ & $\mathrm{TCP}_{\mathrm{BCr}}$ & \\
\hline 1 & $48 \% \rightarrow 70 \%$ & $60 \mathrm{~Gy} \rightarrow 62 \mathrm{~Gy}$ & $95 \%$ & $52 \% \rightarrow 30 \%$ & $56.8 \mathrm{~Gy} \rightarrow 59 \mathrm{~Gy}$ & $95 \%$ & $90 \%$ \\
\hline 2 & \multicolumn{3}{|c|}{ change not needed } & \multicolumn{3}{|c|}{ change not needed } & $94 \%$ \\
\hline 3 & $42 \% \rightarrow 70 \%$ & 60 Gy $\rightarrow 65$ Gy & $96 \%$ & $56 \% \rightarrow 30 \%$ & $55.8 \mathrm{~Gy} \rightarrow 61 \mathrm{~Gy}$ & $95 \%$ & $91 \%$ \\
\hline
\end{tabular}




\begin{tabular}{|c|c|c|c|c|c|c|c|}
\hline \multirow{2}{*}{$\begin{array}{l}\text { Pts } \\
\text { No }\end{array}$} & \multicolumn{3}{|c|}{ SUBVOLUME $A \rightarrow A C r$} & \multicolumn{3}{|c|}{ SUBVOLUME B $\rightarrow \mathrm{BCr}$} & \multirow{2}{*}{$\begin{array}{c}\mathrm{TCP}_{\text {real }} \\
\left(\mathrm{TCP}_{\mathrm{ACr}} \times \mathrm{TCP} \mathrm{P}_{\mathrm{BCr}}\right)\end{array}$} \\
\hline & $V_{A} \rightarrow V_{A R}$ & $\begin{array}{c}\mathrm{NTD}_{\mathrm{A}} \rightarrow \mathrm{NTD}_{\mathrm{AR}} \\
\quad\left(\mathrm{izoGy}_{2.0}\right)\end{array}$ & $\mathrm{TCP}_{\mathrm{ACr}}$ & $\mathrm{V}_{\mathrm{B}} \rightarrow \mathrm{V}_{\mathrm{BR}}$ & $\begin{array}{c}\mathrm{NTD}_{\mathrm{B}} \rightarrow \mathrm{NTD}_{\mathrm{BR}} \\
\left(\mathrm{izoGy}_{2.0}\right)\end{array}$ & $\mathrm{TCP}_{\mathrm{BCr}}$ & \\
\hline 4 & $21 \% \rightarrow 70 \%$ & 60 Gy $\rightarrow 65$ Gy & $96 \%$ & $79 \% \rightarrow 30 \%$ & $56.8 \mathrm{~Gy} \rightarrow 61 \mathrm{~Gy}$ & $94 \%$ & $90 \%$ \\
\hline 5 & $6 \% \rightarrow 70 \%$ & 60 Gy $\rightarrow 65$ Gy & $95 \%$ & $94 \% \rightarrow 30 \%$ & $56.7 \mathrm{~Gy} \rightarrow 61 \mathrm{~Gy}$ & $95 \%$ & $90 \%$ \\
\hline 6 & $79 \%$ & $60 \mathrm{~Gy} \rightarrow 65 \mathrm{~Gy}$ & $95 \%$ & $21 \%$ & $57.4 \mathrm{~Gy} \rightarrow 61 \mathrm{~Gy}$ & $95 \%$ & $90 \%$ \\
\hline 7 & $63 \% \rightarrow 70 \%$ & 60 Gy $\rightarrow 66$ Gy & $95 \%$ & $37 \% \rightarrow 30 \%$ & $55.9 \mathrm{~Gy} \rightarrow 62 \mathrm{~Gy}$ & $95 \%$ & $90 \%$ \\
\hline 8 & \multicolumn{3}{|c|}{ change not needed } & \multicolumn{3}{|c|}{ change not needed } & $92 \%$ \\
\hline 9 & $5 \% \rightarrow 70 \%$ & 66 Gy & $95 \%$ & $95 \% \rightarrow 30 \%$ & 62.4 Gy $\rightarrow 63$ Gy & $94 \%$ & $89 \%$ \\
\hline 10 & $5 \% \rightarrow 70 \%$ & $66 \mathrm{~Gy}$ & $96 \%$ & $95 \% \rightarrow 30 \%$ & 60 Gy $\rightarrow 63$ Gy & $94 \%$ & $90 \%$ \\
\hline 11 & $80 \%$ & 60 Gy $\rightarrow 67$ Gy & $95 \%$ & $20 \%$ & $56.3 \mathrm{~Gy} \rightarrow 63 \mathrm{~Gy}$ & $95 \%$ & $90 \%$ \\
\hline 12 & $4 \% \rightarrow 70 \%$ & $70 \mathrm{~Gy}$ & $97 \%$ & $96 \% \rightarrow 30 \%$ & $60.1 \mathrm{~Gy} \rightarrow 64 \mathrm{~Gy}$ & $94 \%$ & $90 \%$ \\
\hline 13 & $40 \% \rightarrow 70 \%$ & 70 Gy & $97 \%$ & $60 \% \rightarrow 30 \%$ & $56.5 \mathrm{~Gy} \rightarrow 63 \mathrm{~Gy}$ & $97 \%$ & $94 \%$ \\
\hline 14 & $13 \% \rightarrow 70 \%$ & $66 \mathrm{~Gy} \rightarrow 68 \mathrm{~Gy}$ & $95 \%$ & $87 \% \rightarrow 30 \%$ & $63.1 \mathrm{~Gy} \rightarrow 65$ Gy & $94 \%$ & $89 \%$ \\
\hline 15 & $45 \% \rightarrow 80 \%$ & 70 Gy & $97 \%$ & $55 \% \rightarrow 20 \%$ & $64.2 \mathrm{~Gy} \rightarrow 66 \mathrm{~Gy}$ & $99 \%$ & $96 \%$ \\
\hline 16 & $12 \% \rightarrow 70 \%$ & $63 \mathrm{~Gy} \rightarrow 70 \mathrm{~Gy}$ & $96 \%$ & $88 \% \rightarrow 30 \%$ & $60.7 \mathrm{~Gy} \rightarrow 66 \mathrm{~Gy}$ & $97 \%$ & $93 \%$ \\
\hline
\end{tabular}

Besides the absolute number of tumour stem cells, other factors such as hypoxia, clonal radio-resistance, intercellular communication, and repopulation rate may increase inter-tumour or intra-tumour heterogeneity of stem cell density and of the resulting tumour radioresistance. Brenner [22] and Johnson et al. [11] suggested that although some deviations in cellular characteristics of the tumour might modify the volume response to radiation it would unlikely be of crucial importance. Daily fractionation with $2.0 \mathrm{~Gy}$ in all tumours was given which, if at all, might lead to a similar impact on repopulation, which is known to be a major factor causing local recurrences in head and neck cancer. Currently, there is no way to determine heterogeneity of repopulation rates and starting times between tumours. Thus, the contribution of this factor to the findings of our study cannot be properly evaluated. The intra-tumour heterogeneity of tumour stem cell density cannot, at present, be seriously discussed because of the lack of reliable data, however, histopathological studies on stem cell marker distribution may enable us in the future to determine stem cell density in tumours.

Particularly in 3D-IMRT there is a high risk of minor dose inhomogeneity because of the relatively steep gradient of dose within a narrow distance from the centre of the tumour. Tome and Fowler [7, 8], Withers [9, 10] and other authors discussed in detail the physical and clinical aspects of "cold spots" and "cold doses". Whereas GTV can be precisely contoured using radiological images, CTV and PTV can only be individually surmised based on the experience of the radiation oncologist because there is no chance to image small conglomerates of tumour (stem) cells outside the GTV. Therefore we focused on underdosed cold spots within the GTV. At the edge of the SVB the dose may even be a bit lower, but we used an average value to simplify our model. With constant number of fractions, the dose per fraction is also reduced. To compare biological effectiveness of the total doses in both SVA and SVB, mean total doses $\left(\mathrm{NTD}_{\mathrm{s}}\right.$ ) in the SVB were normalised to the dose given in 2.0 Gy fractions using the L-Q model with = $10 \mathrm{~Gy}$ and listed as NTD IzoGy . $^{2}$

The relationship between planned and delivered $\mathrm{NTD}_{S}$ for SVA and SVB is presented in table II. The results show that the size of the SVA which received $100 \%$ of the planned total dose ranged from $5 \%$ to $82 \%$ but the mean TD in SVA was high enough to correspond with high $\mathrm{TCP}_{\mathrm{A}^{\prime}}$ except case no.11 for which the planned TD was too low to eradicate the SVA. For the SVB, the situation was worse. In 11 cases, the SVB was larger than the SVA. The real TCP values were estimated by multiplying $\mathrm{TCP}_{\mathrm{A}}$ and $\mathrm{TCP}_{\mathrm{B}}$ calculated for SVA and SVB. The real $T C P_{R L}$ values significantly differ from the planned $T C P_{P L}$ values. All local recurrences occurred in those cases in which a significantly reduced real $T C P_{R L}$ was calculated.

Our results in the present study support the suggestions of other authors that the biological impact of heterogeneous dose distribution and dose deficit in tumour subvolumes depends not only on the dose deficit but also on the extent of the cold spot(s). Tome, Fowler, Withers [7-10] and other authors postulated that a cold spot of $20-40 \%$ of the target volume underdosed by $10 \%$ of the prescribed TD would cause the loss in TCP by about 15\% or more. Our observations are in agreement with those theoretical predictions. Yet, we also agree with Tome and Fowler [7, 8] and Goitein and Niemierko [19] that a significant decrease in the TCP depends steeply on dose even for small cold volumes, and that such a deficit cannot be rectified by boosting the dose to the relatively large volume of the PTV.

It is obvious that using IMRT and other 3D-conformal techniques, some dose inhomogeneity in the GTV is unavoidable. The efficacy of these radiotherapy techniques cannot only be dealt with on the basis of physical parameters alone, disregarding radiobiological principles [10]. TCP should be 
considered as a function not only of dose but also of the initial number of tumour stem cells, indirectly expressed by tumour subvolumes but not by tumour stages. The treatment outcome is strongly influenced by unaccounted differences in a spatial dose distribution. The hazard of cold spots has been clearly documented and intuitively, even a cubic millimetre of receiving a low dose may lead to recurrence. Such a risk significantly increases when the size of a cold spot enlarges from millimetres to cubic centimetres. It must be estimated a priori as an essential part of treatment planning. Our model involves the simple assumption of constant stem cell density, and uniform dose distribution in each of the two subvolumes (more than two SV can also be analysed). This model should be taken only as example of what might occur in practice.

Whereas complex TCP equations defined by Tome [7, 8] and Goitein and Niemierko [19] may be useful for mathematically sophisticated analyses, they are useless for daily planning by radiation oncologists. Our proposition of TCP estimation (i.e. our equation no. 6) is simple and can easily be used even by a mathematically inexperienced radiation oncologist, and it takes only about one minute using a simple calculator with Ln and Log functions.

The unacceptable discrepancies between the planned

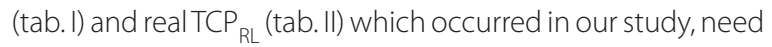
re-planning procedures with the aim of enlarging the D100 subvolume (SVA) and minimising the size of the underdosed cold subvolume (SVB) as much as possible. Examples of such correction of the IMRT planning are shown in table III.

\section{Conclusions}

In 3D-IMRT and other conformal radiotherapy techniques, inhomogeneous dose distributions are unavoidable. Therefore the hazard of underdosed cold spot(s) within the target volume (at least GTV) should be accounted for. The efficacy of these radiotherapy techniques expressed by local tumour probability cannot be considered based on physical parameters alone, disregarding radiobiological principles. Tumour volume (but not tumour stage) is an appropriate though approximate measure of initial number of tumour stem cells which is the most relevant predictor of the TCP. The biological impact of any dose deficit in the cold spot(s) on the TCP depends not only on the magnitude of the deficit but on the size of the cold spot subvolume. Instead of the $95 \%$ isodose criterion, mapping $V_{100}$ within the target receiving $100 \%$ of the planned dose is recommended, which should be as large as possible, minimising the biological impact of the underdosed cold subvolume(s). The real TCP $R$ is the product of the $T C P_{A}$ for the $V_{100}$ and TCP for the cold subvolume. Any serious discrepancy between the real $T C P_{R \mid}$ and the planned $T C P_{P L}$ requires precise re-planning and correction of dose distribution within GTV subvolumes.

This paper is dedicated to the memory of Rod Withers who initiated the concept of the present work.
Conflict of interest: none declared

\section{Bogusław Maciejewski}

M. Skłodowska-Curie National Research Institute of Oncology,

Gliwice Branch

Department Radiotherapy

Wybrzeże Armii Krajowej 15

44-102 Gliwice, Poland

e-mail:boguslaw.maciejewski@io.gliwice.pl

\section{Received: 22 May 2020}

Accepted: 2 Jun 2020

\section{References}

1. Kummermehr J, Trott KR. Tumour stem cells. Stem Cells. 1997: 363-399, doi: 10.1016/b978-012563455-7/50013-1.

2. Suit HD, Shalek RJ, Wette R. Radiation Response of C3H Mouse Mammary Carcinoma Evaluated in Terms of Cellular Radiation Sensitivity. In: Cellular Radiation Biology. Williams and Wilkins, Baltimore 1965.

3. Guttenberger R, Kummermehr J, Wang J et al. 38 Ann Meeting Radiat Res Soc, abst. Cn-5 New Orleans, 1990.

4. Maciejewski BA, Skates S, Zajusz A, et al. Importance of tumor size and repopulation for radiocurability of skin cancer. Neoplasma. 1993;40(1): 51-54, indexed in Pubmed: 8350948.

5. Dubben HH, Thames HD, Beck-Bornholdt HP. Tumor volume: a basic and specific response predictor in radiotherapy. Radiother Oncol. 1998; 47(2): 167-174, doi: 10.1016/s0167-8140(97)00215-6, indexed in Pubmed: 9683365.

6. Magee BJ, Logue JP, Swindell R, et al. Tumour size as a prognostic factor in carcinoma of the cervix: assessment by transrectal ultrasound. $\mathrm{Br} J$ Radiol. 1991; 64(765): 812-815, doi: 10.1259/0007-1285-64-765-812, indexed in Pubmed: 1913045.

7. Tome WA, Fowler JF. Selective boosting of Tumour subvolumes. Int J Radiat Oncol Biol Phys. 2000; 47: 1137-1143.

8. Tomé WA, Fowler JF. On cold spots in tumor subvolumes. Med Phys. 2002; 29(7): 1590-1598, doi: 10.1118/1.1485060, indexed in Pubmed: 12148742.

9. Perez C, Brady L. Principles and Practice of Radiation Oncology. Pediatr Hematol Oncol. 1999; 21(6): 560, doi: 10.1097/00043426-19991100000025.

10. Withers HR. Biological aspects of conformal therapy. Acta Oncol. 2000; 39(5): 569-577, doi: 10.1080/028418600750013258, indexed in Pubmed: 11093365.

11. Johnson $\mathrm{C}$, Thames $\mathrm{H}$, Huang $\mathrm{D}$, et al. The tumor voluem and clonogen number relationship: Tumor control predictions based upon tumor volume estimates derived from computed tomography. Int J Radiat Oncol Biol Phys . 1995; 33(2): 281-287, doi: 10.1016/0360-3016(95)00119-j.

12. Deasy J.Tumour control probability model for non-uniform dose distributions. In: Paliwal B, Fowler JF, Hubert D, Mehta MP. ed. Volume and Kinetics in Tumour Control and Normal Tissue Complications. The Fifth Madison International Conference on Time, Dose and Fractionation. AIP, New York 1997: 65-85.

13. KnöösT, Kristensen I, Nilsson P. Volumetric and dosimetric evaluation of radiation treatment plans: radiation conformity index. Int J Radiat Oncol Biol Phys. 1998; 42(5): 1169-1176, doi: 10.1016/s0360-3016(98)00239-9, indexed in Pubmed: 9869245.

14. Mayo CS, Ding L, Addesa A, et al. Initial experience with volumetric IMRT (RapidArc) for intracranial stereotactic radiosurgery. Int J Radiat Oncol Biol Phys. 2010; 78(5): 1457-1466, doi: 10.1016/j.jirobp.2009.10.005, indexed in Pubmed: 20207494.

15. Guerrero Urbano MT, Clark CH, Kong C, et al. Target volume definition for head and neck intensity modulated radiotherapy: pre-clinical evaluation of PARSPORT trial guidelines. Clin Oncol (R Coll Radiol). 2007; 19(8): 604-613, doi: 10.1016/j.clon.2007.07.001, indexed in Pubmed: 17706404.

16. Bentzen SM. Quantitative clinical radiobiology. Acta Oncol. 1993; 32(3): 259-275, doi: 10.3109/02841869309093594, indexed in Pubmed: 8323764.

17. Yaes RJ. Tumor heterogeneity, tumor size, and radioresistance. Int J Radiat Oncol Biol Phys. 1989; 17(5): 993-1005, doi: 10.1016/03603016(89)90147-8, indexed in Pubmed: 2808062.

18. Harel D, Mills SD, Kwakkenbos L, et al. SPIN Investigators, members of the ISUP Renal Tumor Panel. Weighted kappa: nominal scale agreement 
with provision for scaled disagreement or partial credit. Psychol Bull. 1968; 70(4): 213-220, doi: 10.1037/h0026256, indexed in Pubmed: 19673146.

19. Goitein M, Niemierko A. Intensity modulated therapy and inhomogeneous dose to the tumor: A note of caution. Int J Radiat Oncol Biol Phys. 1996; 36(2): 519-522, doi: 10.1016/s0360-3016(96)00348-3.

20. Muralikrishnan B, Raja J. Least-squares best fit line and plane. In: Muralikrishnan B, Raja J. ed. Computational Surface and Roundness Metrology. Springer Verlag London Ltd, London 2009: 121-130.
21. Hartigan JA, Wong MA. Algorithm AS 136: A K-Means Clustering Algorithm. Applied Statistics. 1979; 28(1): 100, doi: 10.2307/2346830.

22. Brenner DJ. Dose, volume, and tumor-control predictions in radiotherapy. Int J Radiat Oncol Biol Phys. 1993; 26(1): 171-179, doi: 10.1016/03603016(93)90189-3, indexed in Pubmed: 8482624 